\title{
Recombinant adeno-associated virus-mediated hu- man kallikrein gene therapy protects against hyper- tensive target organ injuries through inhibiting cell apoptosis
}

\author{
Jiang-tao YAN", Tao WANG", Dao-wen WANG* \\ Department of Internal Medicine and the Institute of Hypertension, Tongji Hospital, Tongji Medical College of Huazhong University of \\ Science and Technology, Wuhan 430030, China
}

\begin{abstract}
Aim: Overexpression of human tissue kallikrein (HK), mediated by recombinant adeno-associated virus ( $\mathrm{rAAV}$ ), decreased blood pressure in spontaneous hypertensive rats (SHRs) and reduced injury to the heart, aorta and kidney. In this study, we used both an in vivo animal model and in vitro cell culture system to investigate whether rAAV-mediated HK gene therapy protects against organ damage by inhibiting cell apoptosis.

Methods: rAAV encoding HK (rAAV-HK) or LacZ (rAAV-lacZ) were delivered as a control to spontaneously hypertensive rats (SHRs) and cultured human embryonic kidney (HEK) 293 cells.

Results: Treatment with rAAV-HK decreased cell apoptosis in the target organs of SHRs and also inhibited lipopolysaccharide (LPS)-induced HEK 293 apoptosis. The rAAV-HK delivery system also increased the levels of apoptosis-inhibiting proteins bcl-2 and bcl- $\mathrm{X}_{\mathrm{L}}$, and decreased the level of Bax and the activity of caspase 3, two promoters of apoptosis. In addition to its role in the inhibition of apoptosis, rAAV-HK also activated the cell survival and proliferation signaling pathways ERK1/2 and PI3K/AKT.

Conclusion: rAAV-mediated HK gene delivery has multiple therapeutic possibilities for treating hypertension, not only by decreasing blood pressure, but also by directly inhibiting end-organ damage.
\end{abstract}

Keywords: kallikrein; recombinant adeno-associated virus; gene therapy; hypertension; apoptosis; target organs injuries

Acta Pharmacologica Sinica (2009) 30: 1253-1261; doi: 10.1038/aps.2009.114; published online 17 August 2009

\section{Introduction}

Hypertension is a chronic disease affecting approximately 1 billion individuals worldwide and causing approximately 7.1 million deaths per year ${ }^{[1]}$. Hypertension damages major organs, eventually leading to heart failure, atherosclerosis and kidney diseases. Several clinical trials have demonstrated that although some special outcomes differ between the classes, the different drugs available for lowering blood pressure have similar mechanisms of cardiovascular prevention ${ }^{[1]}$.

Gene therapy may provide long-term control of blood pressure for hypertensive patients ${ }^{[2]}$. Recombinant adeno-associated virus (rAAV) is an appealing vector for gene therapy protocols ${ }^{[3]}$, possibly mediating stable transduction and long-term expression of target genes in vivo. Data accumulated from

\footnotetext{
\# These two authors contributed equally to this work.

* To whom correspondence should be addressed.

E-mail dwwang@tjh.tjmu.edu.cn

Received 2009-03-03 Accepted 2009-06-29
}

thousands of animal studies and hundreds of human patients have demonstrated that rAAV vectors can be effectively and safely used for gene therapy ${ }^{[4]}$.

The kallikrein/kinin system (KKS) plays an important role in cardiovascular disease and prevention. Tissue kallikrein cleaves the low molecular weight kininogen substrate to produce the kinin peptide, which binds to bradykinin B2 receptor to trigger a broad spectrum of biological effects, such as vasodilation, inflammation, pain, and cell proliferation ${ }^{[5]}$. The kallikrein gene has become a popular target gene for the treatment of cardiovascular diseases.

Our previous studies showed that overexpression of human tissue kallikrein (HK) mediated by rAAV caused a long-term decrease in blood pressure in spontaneous hypertensive rats (SHRs) and high salt-induced hypertensive rats and lessened injuries to the heart, aorta and kidney ${ }^{[6-8]}$. We hypothesized that in addition to its blood pressure-lowering effects, KKS could prevent end-organ injury by inhibiting apoptosis. In this study, we delivered rAAV encoding HK (rAAV-HK) to 
SHRs and cultured human embryonic kidney 293 cells, using rAAV encoding LacZ (rAAV-lacZ) as a control, to determine whether overexpression of HK can block apoptosis.

\section{Materials and methods}

Production and measurement of titers of rAAV serotype- 2 vectors The rAAV vector plasmid pXXUF 1 , the rAAV plasmid containing a reporter gene, pdxII-LacZ (control), the packaging plasmid pXX2 and the adenovirus helper plasmid pXX6 have been described previously ${ }^{[9]}$. The pXXUF1 vector contains two inverse terminal repeats, a cytomegalovirus promoter (CMV) and a poly-A tail. An 860-bp HK fragment (NotI/NotI) containing an open reading frame was subcloned into $\mathrm{pXXUF}_{1}$ downstream of CMV to establish the pUF1-HK plasmid. The rAAV $\cdot H K$ and $\mathrm{rAAV}$ LacZ viruses were produced as described previously ${ }^{[6]}$. The titer of rAAV-HK and rAAV-lacZ was determined by dot blot hybridization.

\section{Animal treatment and gene delivery}

Animal treatment and gene delivery were described previously ${ }^{[6]}$. Twelve three-month-old male SHRs weighing 250-280 g were randomly divided into two groups (six rats in each group). A single injection of rAAV-HK (about $1 \times 10^{11}$ transducing units in $1 \mathrm{~mL}$ of saline solution) into the tail of the SHRs was administered in the treatment group. For the control group, the SHRs were injected with rAAV-lacZ. Nineteen weeks after rAAV delivery, all animals were sacrificed using an intraperitoneal injection of pentobarbital at a dose of 50 $\mathrm{mg} / \mathrm{kg}$ body weight. Part of the heart, kidney and aorta was collected from each rat in liquid nitrogen and stored at $-80{ }^{\circ} \mathrm{C}$. The other part of each organ was embedded in paraffin and cut into sections $(4 \mu \mathrm{m})$ for the TdT-mediated dUTP nick endlabeling (TUNEL) assay.

All animal experimental protocols were approved by the Institutional Animal Research Committee of Tongji Medical College and carried out according to the guidelines of the National Institutes of Health.

\section{TdT-mediated dUTP nick end-labeling (TUNEL) assay}

To investigate apoptosis in the organs of hypertensive rats, we conducted a TdT-mediated dUTP nick end-labeling (TUNEL) assay on paraffin-embedded tissue sections of the heart, kidney and aorta of the SHRs. The TUNEL assay kit was obtained from R\&D Systems (Minneapolis, USA), and the in situ apoptosis analysis was performed according to the manufacturer's instructions. The TUNEL-positive (apoptotic) cells were counted in 10 microscopic fields of each tissue section.

\section{Treatments of human embryonic kidney 293 cells}

Human embryonic kidney (HEK) 293 cells, which express the bradykinin B2 receptor, were cultured in DMEM containing $10 \% \mathrm{FBS}^{[10]}$. Apoptosis was induced by incubating the HEK 293 cells with $200 \mu \mathrm{mol} / \mathrm{L}$ LPS in non-FBS DMEM medium for $24 \mathrm{~h}$. In order to investigate the effect of HK overexpression on apoptosis, the HEK 293 cells were treated with $1 \times 10^{8}$ pfu. rAAV-HK or rAAV-lacZ (control) for $3 \mathrm{~d}$ before LPS-induced apoptosis. To demonstrate the effect of kinin on cell apoptosis, HEK 293 cells were treated with human bradykinin $\left(1 \times 10^{-6}\right.$ $\mathrm{mmol} / \mathrm{L}$ ) or bradykinin+Hoe140 (a Bradykinin B2 receptor inhibitor, $1 \times 10^{-4} \mathrm{mmol} / \mathrm{L}$ ) for $2 \mathrm{~h}$ before LPS-induced apoptosis. The positive control was only treated with LPS in nonFBS DMEM medium, and the negative control received no treatment or LPS. Apoptotic responses were assessed by three independent methods, including Acridine orange/Ethidium Bromide staining analysis, annexin V and PI FACS analysis, and caspase 3 activity assay, as described below.

\section{Acridine orange/Ethidium bromide (AO/EB) staining analysis}

To detect nuclear DNA condensation, cell nuclear staining (AO/EB staining) was performed. A dye mixture containing $100 \mu \mathrm{g} / \mathrm{mL}$ of both acridine orange and ethidium bromide $(\mathrm{AO} / \mathrm{EB})$ was added to the cell culture dish at a final concentration of $3 \times 10^{5} / \mathrm{mL}$. Cells with intact nuclear membranes stained green, while nuclei that had lost membrane integrity stained orange. Apoptotic cells could be distinguished from nonapoptotic cells on the basis of the presence of nuclear condensation and fragmentation.

\section{Annexin V and PI FACS analysis}

HEK 293 apoptosis was assayed by annexin $V$ and propidium iodide (PI) staining and analyzed by fluorescence-activated cell sorting (FACSVantage ${ }^{\mathrm{TM}}$, BD Biosciences, USA).

\section{Caspase 3 activity assay}

To determine caspase 3 activity, HEK 293 cells were washed twice with ice-cold phosphate-buffered saline and then lysed in the Cell Lysis Buffer provided in the Caspase 3 Colorimetric Assay Kit (R\&D Systems). The cell lysates were concentrated with Centricons (mol wt cut-off 3 000; Millipore) to obtain protein concentrations of $2-4 \mathrm{mg} / \mathrm{mL}$. The enzymatic reaction for caspase 3 activity was carried out using the p-nitroanilide-conjugated DEVD peptide (DEVD-pNA) substrate, as described by the manufacturer. The results were quantitated by spectrophotometry at a wavelength of $405 \mathrm{~nm}$.

\section{Western blot analysis}

Total tissue protein was extracted from SHR tissues and cultured HEK 293 cells using TRIzol reagent (Invitrogen Life Technologies) and protein concentrations were detected by the Bradford method. Protein samples ( $20 \mu \mathrm{g}$ per lane) were separated by $10 \%$ SDS/PAGE, electrophoretically transferred onto PVDF membranes, and the membranes were blocked for $2 \mathrm{~h}$ at room temperature with $5 \%$ nonfat dried milk in $10 \mathrm{mmol} /$ L Tris- $\mathrm{Cl}$ (pH 7.5), $100 \mathrm{mmol} / \mathrm{L} \mathrm{NaCl}$, and $0.1 \%$ Tween-20 (TBS-T). The membranes were then incubated overnight at $4{ }^{\circ} \mathrm{C}$ with rabbit anti- $\beta$-actin polyclonal antibody, followed by a $2 \mathrm{~h}$ incubation at room temperature with goat anti-rabbit IgG conjugated to horseradish peroxidase. For some experiments, rabbit anti-PI3K (1:500 dilution), rabbit anti-AKT (1: 1000 dilution), rabbit anti-phospho-AKT(1: 1000 dilution), rabbit antiERK1/2 (1: 1000 dilution), rabbit anti-Phospho-ERK1/2 (1: 1000 dilution), rabbit anti-bcl-2 (1:1000 dilution), rabbit anti- 
bcl- $x_{\mathrm{L}}$ (1: 1000 dilution) or mouse anti-bax (1: 1000 dilution) was used instead of the anti- $\beta$-actin antibody.

\section{Statistical analysis}

Data are expressed as means \pm SDs. Differences were analyzed for significance by one-way repeated-measures ANOVA and further analyzed with the use of the Newman-Keuls test for multiple comparisons between treatment groups. The results were considered significant at $P<0.05$.

\section{Results}

Kallikrein gene delivery attenuates cell apoptosis in target organs of SHRs

First, we used the TUNEL staining assay to detect apoptotic cells in target organs of SHRs that were treated as described above. Our data showed that the heart, kidney and aorta of rAAV-HK-treated SHRs had significantly fewer TUNELpositive cells than rAAV-LacZ-treated SHRs (Figure 1A), and kallikrein gene delivery significantly reduced the ratio of TUNEL-positive cells to total tissue cells compared with the control group $(n=6, P<0.01)$ (Figure $1 \mathrm{~B})$. These results suggest that kallikrein delivery attenuates cell apoptosis in the target organs of SHRs.

Kallikrein gene delivery significantly activates ERK1/2 and PI3K/ AKT signaling pathways in SHRs

We also examined whether HK gene delivery could change the balance between cell death and survival by altering critical cell proliferation signaling pathways. Thus, we examined the effects of rAAV-HK on the PI3K/AKT and ERK1/2 signaling pathways in SHRs using Western blotting. The protein levels of PI3K, phosph-AKT and phosph-ERK1/2 were significantly increased in rAAV-HK-treated SHRs compared with those treated with rAAV-lacZ (Figure 2). These increased protein levels indicated that overexpression of kallikrein stimulated phosphorylation of ERK1/2 and PI3K/AKT signaling molecules in the target organs of SHRs, which might also contribute to the anti-apoptotic effects of kallikrein gene delivery in SHRs.

Kallikrein gene delivery inhibits apoptosis by affecting the bcl-2 protein family

In order to understand the mechanisms underlying the antiapoptotic effects of HK gene delivery on the target organs of SHRs, we examined the expression levels of different genes in the bcl-2 protein family using Western blotting. Kallikrein gene delivery significantly increased the protein levels of bcl-2 and bcl-xl, two known anti-apoptosis proteins, and decreased the protein level of Bax, a pro-apoptosis protein (Figure 3). These observations suggested that the overexpression of kallikrein gene inhibited hypertension-induced cell apoptosis by modulating the expression of bcl-2 protein family members.

Kallikrein/kinin system inhibits HEK 293 LPS-induced apoptosis Having determined the protective effects of kallikrein against apoptosis in vivo, we then sought to confirm these effects in an in vitro cell culture system. The effects of the kallikrein/
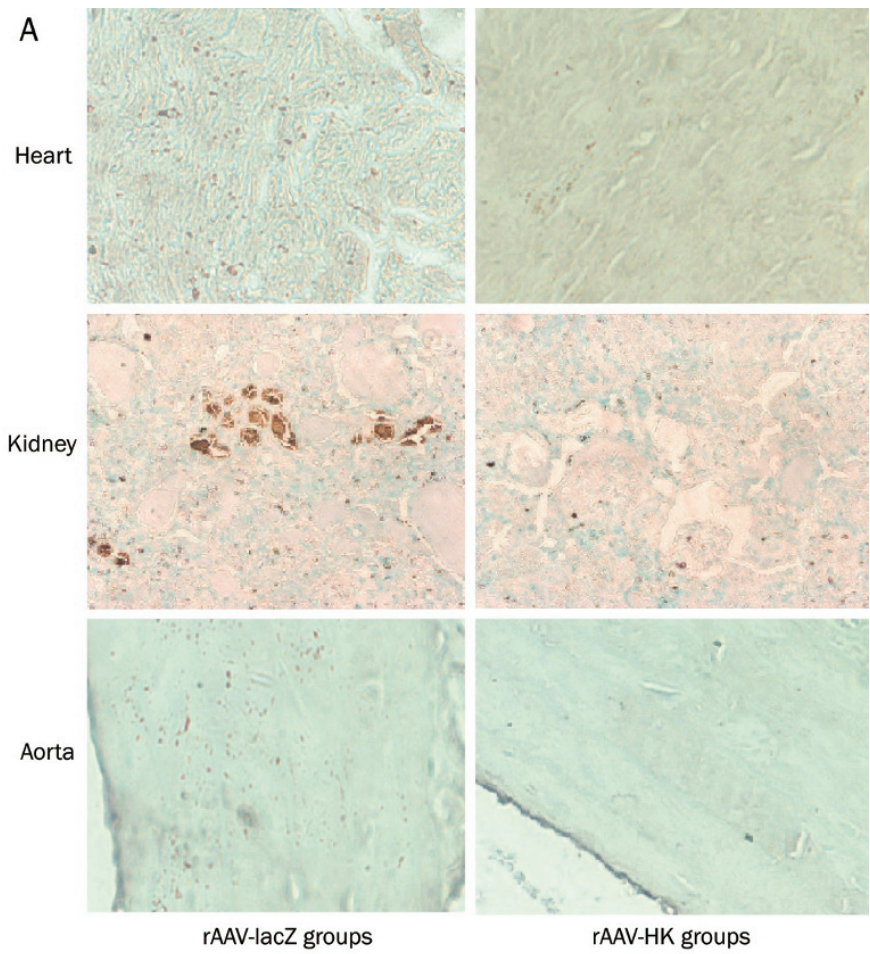

B

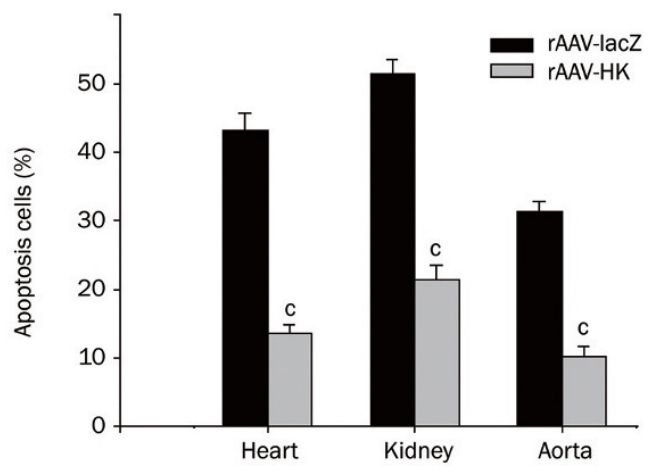

Figure 1. Kallikrein gene delivery attenuates apoptosis in organs of SHRs. TUNEL-positive (apoptotic) cells were counted in 10 microscopic fields for each tissue section. TUNEL-positive staining of all tissues was significantly reduced in the rAAV-HK-treated SHRs compared with the rAAV-LacZ-treated SHRs (Figure 1A). As seen in the histogram, Kallikrein gene delivery significantly reduced the ratio of TUNEL-positive cells to total tissue cells compared with the control group $\left(n=6,{ }^{c} P<0.01\right.$ vs rAAV-LacZ group, Figure 1B).

kinin system on HEK 293 cell apoptosis were detected by acridine orange/ethidium bromide staining and flow cytometry (double staining with annexin V and PI). rAAV-HK and Kinin treated HEK 293 cells had markedly reduced LPS-induced apoptosis $(P<0.05)$, while rAAV-lacZ had no effect on LPSinduced apoptosis. Hoe 140, a B2 receptor inhibitor, attenuated the anti-apoptotic effect of kinin (Figures 4, 5). These data indicate that the kallikrein/kinin system has a direct antiapoptotic effect by activating the $\mathrm{B} 2$ receptor. 

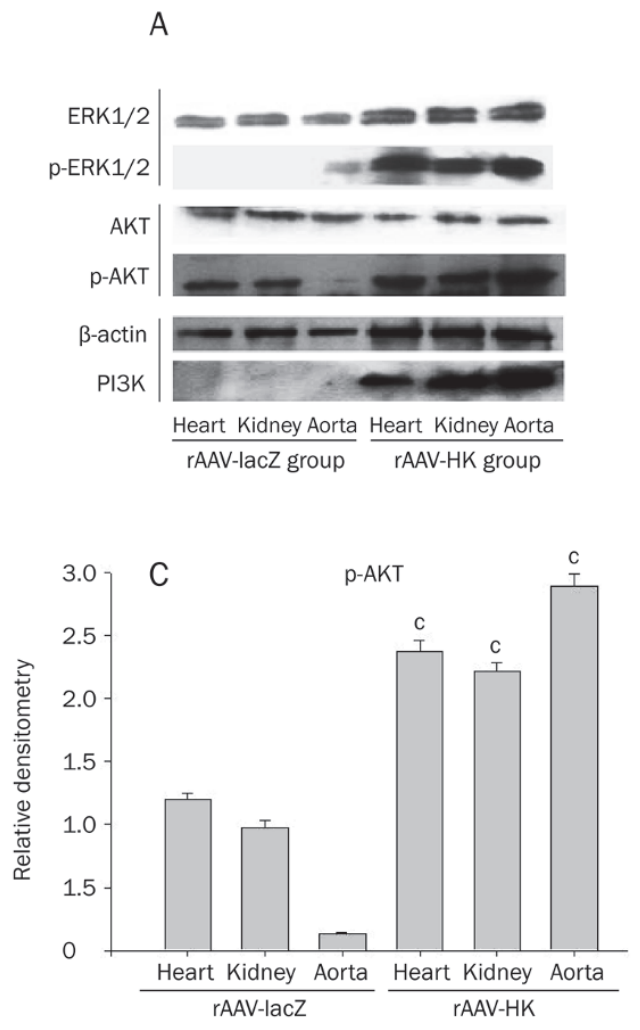

A

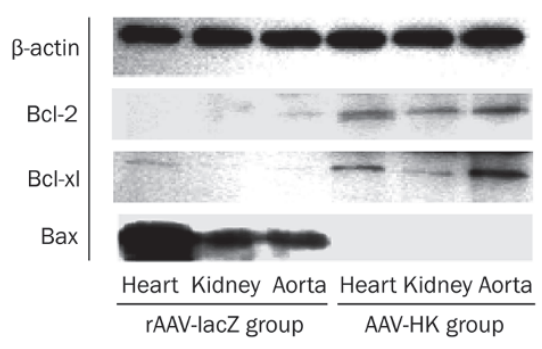

C

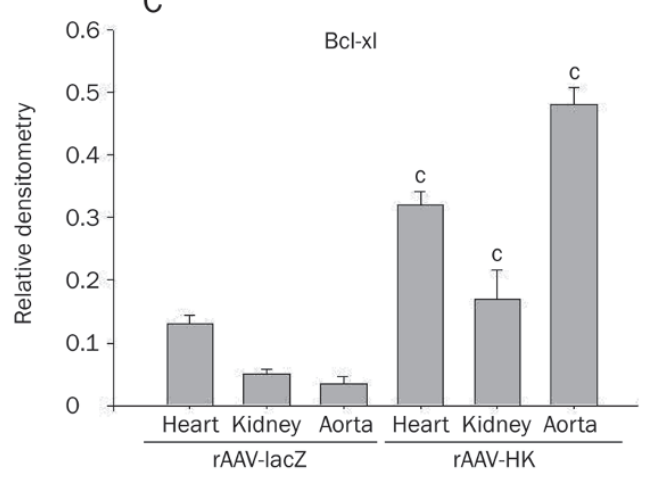

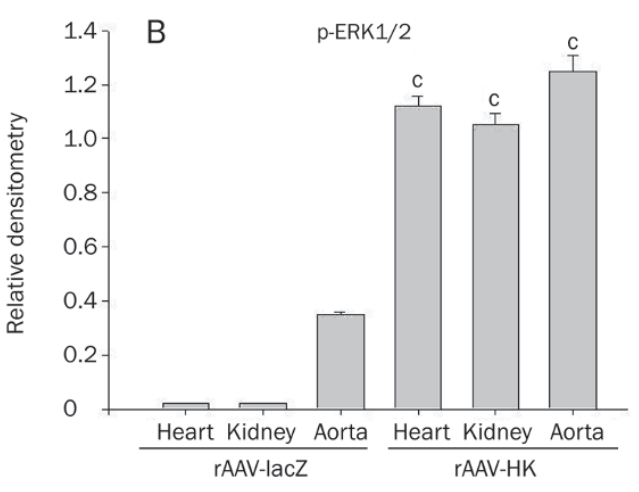
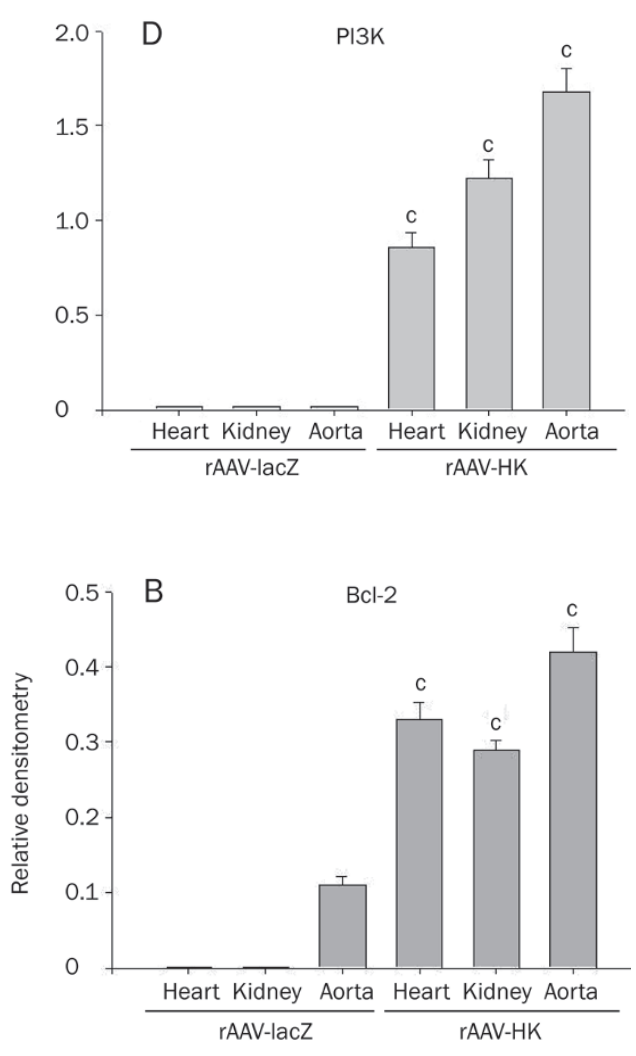

D

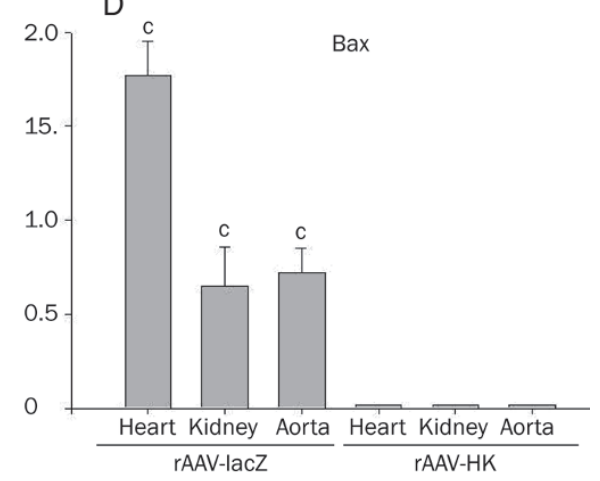

Figure 2. Kallikrein gene delivery significantly activates cell proliferation signaling pathways in SHRs. The protein levels of phosph-ERK1/2, phosph-AKT, and PI3K were detected by Western blotting (A), and the expression levels, as determined by densitometry, were normalized to total ERK1/2, AKT, and $\beta$-actin (B, C, D). The histogram displays the significant increase of $\mathrm{p}$-ERK1/2, p-AKT, and PI3K in rAAV-HK-treated SHR compared with rAAV-lacZ control group. ${ }^{c} P<0.01$
Figure 3. The kallikrein/kinin system inhibits apoptosis by modulating the bcl- 2 protein family. Protein expression levels in the tissue samples were detected by Western blot analysis (A). Blots were scanned and the relative expression levels, as determined by densitometry, were normalized to total $\beta$-actin as shown in the histogram (B, C, D). Kallikrein gene delivery significantly increased the protein levels of $\mathrm{bcl}-2$, and $\mathrm{bcl}$ $x_{L}$ two anti-apoptotic proteins, and decreased the protein level of Bax, a pro-apoptotic protein. ${ }^{c} P<0.01$ vs rAAV-LacZ group.
Kallikrein/kinin system reduces caspase 3 activity

Caspase 3 is a central executor of apoptosis signaling. We tested whether rAAV-HK delivery could alter downstream signaling of the apoptosis pathway by affecting caspase 3 activity. The results of the caspase 3 activity analysis showed that with non-FBS medium, the activity of caspase 3 increased 
A
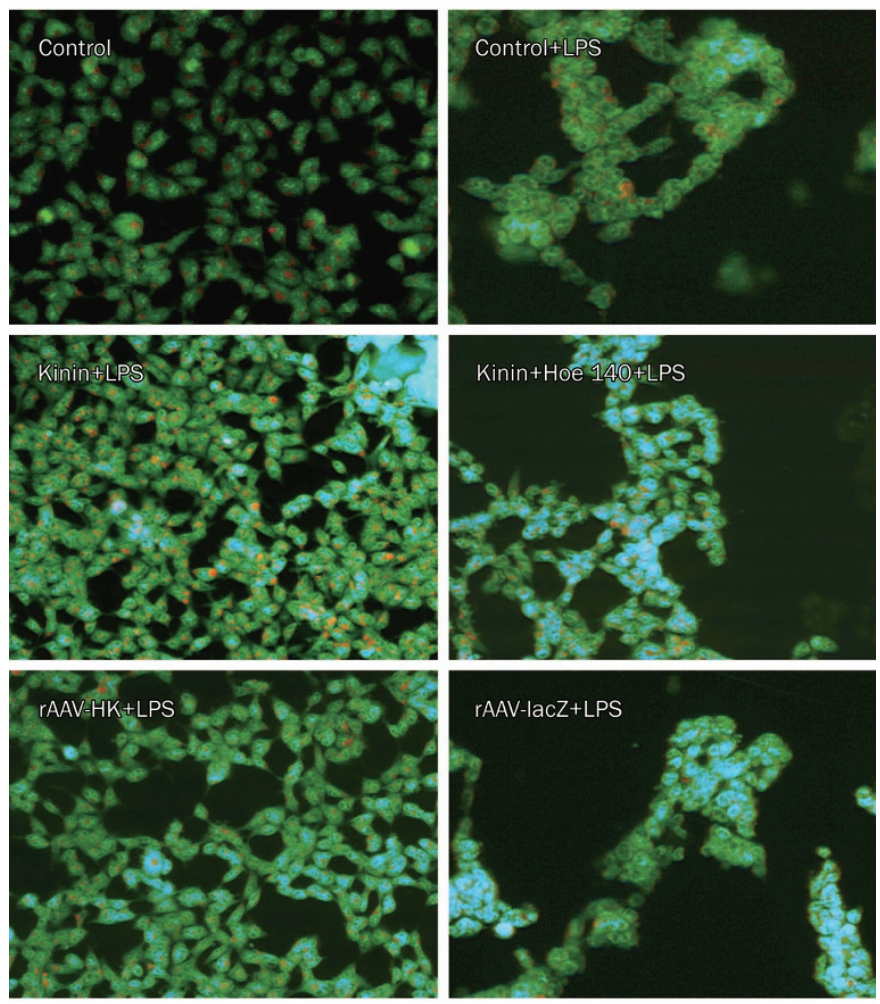

B

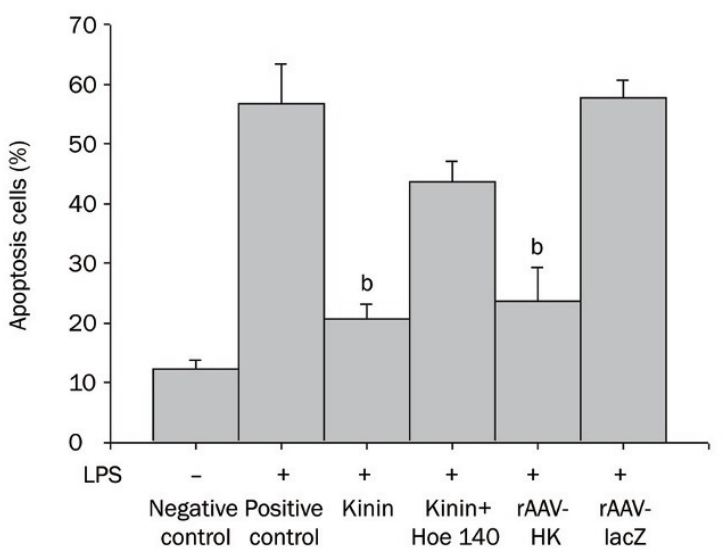

Figure 4. The kallikrein/kinin system reduces the ratio of apoptotic-tolive cells after apoptosis induction. HEK 293 cells were examined under a fluorescent microscope after acridine orange/ethidium bromide staining (A). Bright green cells with condensed chromatin are live apoptotic cells. Bright orange cells with condensed chromatin are dead apoptotic cells. Fainter green and orange cells with diffuse chromatin are non-apoptotic live and dead cells, respectively. The results showed that apoptosis increased when cells were exposed to LPS in non-FBS medium (positive control group) compared to cells in the same medium without LPS (negative control group). Treatment with kinin or rAAV-HK markedly reduced LPSinduced HEK 293 apoptosis $(P<0.05)$, while rAAV-lacZ had no effect on apoptosis. Hoe 140, a B2 receptor inhibitor, attenuated the effects of kinin on apoptosis(B). ${ }^{\mathrm{c}} \mathrm{P}<0.01$ vs rAAV-LacZ.

significantly upon LPS exposure compared with the negative control group. Treating cells with rAAV-HK and kinin, but
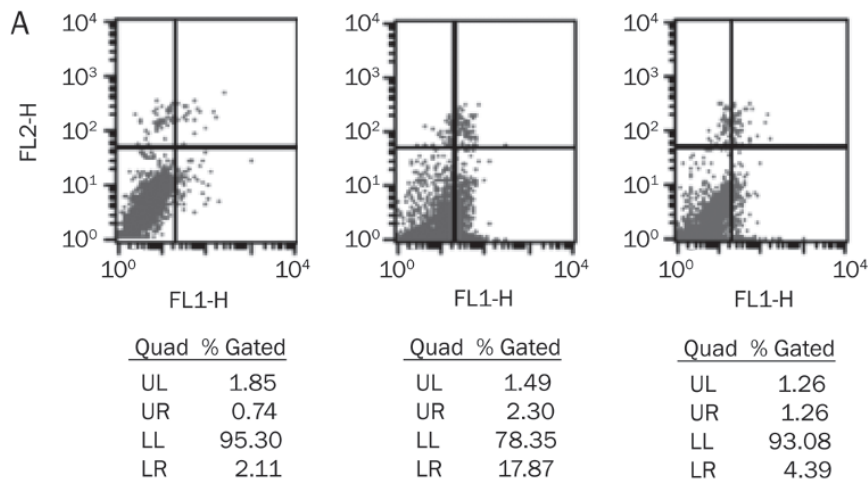

LPS

Negative control
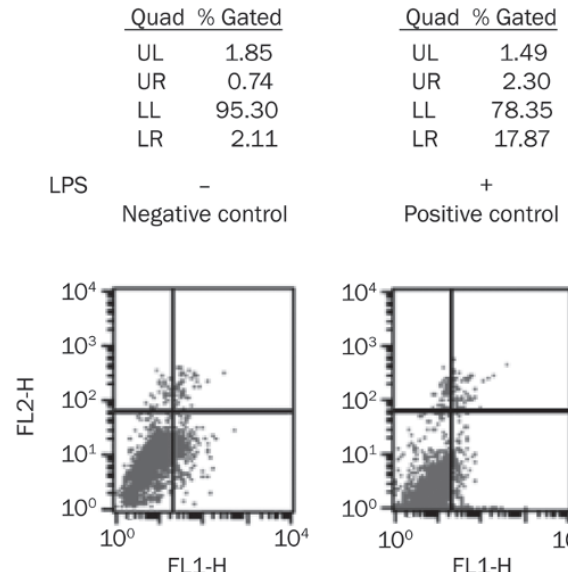

$+$

Positive control
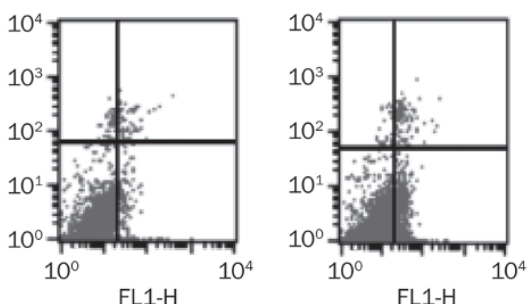

Quad \% Gated

$\begin{array}{lr}\text { UL } & 1.46 \\ \text { UR } & 2.09 \\ \text { LL } & 96.98 \\ \text { LR } & 9.47\end{array}$

LPS

Kinin+Hoe 140

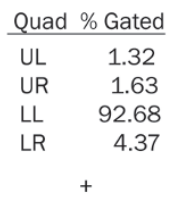

rAAV-HK

Quad \% Gated

UR $\quad 1.26$

LL $\quad 93.08$

LR $\quad 4.39$

$+$

Kinin

Quad \% Gated

$\begin{array}{lr}\text { UL } & 0.91 \\ \text { UR } & 2.68 \\ \text { LL } & 81.12 \\ \text { LR } & 15.29\end{array}$

rAAV-lacZ

B

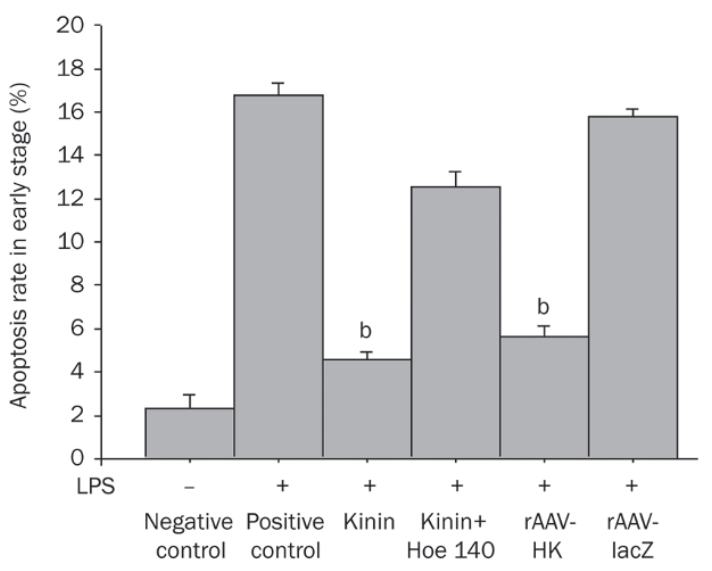

Figure 5. The kallikrein/kinin system reduces the ratio of apoptoticto-live cells after apoptosis induction. Annexin V and PI FACS analysis showed that the early-stage apoptotic rate (positive for annexin $V$ and negative for $\mathrm{PI}$ ) increased in non-FBS medium with LPS (positive control group) compared to that in medium without LPS (negative control group). Treatment with kinin and rAAV-HK markedly decreased apoptosis compared to the positive control group, the kinin+Hoe140 group and the rAAV-lacZ group ( $\left.{ }^{\mathrm{b}} P<0.05\right)$, and these cells had no obvious differences compared to the negative control group.

not rAAV-lacZ, inhibited the effect of LPS on caspase 3 activity $(P<0.05)$. Kinin+Hoe140 did not affect caspase 3 activity after 


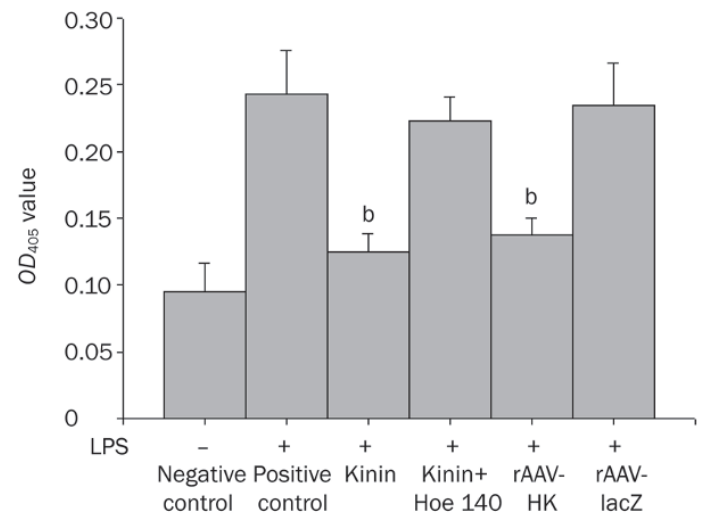

Figure 6. The kallikrein/kinin system reduces apoptosis by inhibiting the caspase-related signaling pathways. In non-FBS medium, LPS exposure significantly increased the activity of caspase 3 compared to the negative control group. Treatment with rAAV-HK and kinin, but not rAAV-lacZ, inhibited the effects of LPS on caspase 3 activity $(P<0.05)$. Kinin+Hoe140 had no effects on the LPS-induced increase in caspase 3 activity.

LPS exposure (Figure 6). These data suggest that overexpression of the kallikrein gene inhibits LPS-induced apoptosis by modulating the activity of caspase 3 through activation of the B2 receptor.

Kallikrein/kinin system activates ERK1/2 and PI3K/AKT signaling pathways in HEK 293 cells

We also confirmed that the kallikrein/kinin system activates the PI3K/AKT and ERK1/2 signaling pathways in HEK 293 cells using Western blotting. Expression of PI3K, phosphoAKT and phospho-ERK1/2 decreased when HEK 293 cells were exposed to LPS in non-FBS medium. Treatment with rAAV-HK and kinin, but not rAAV-lacZ, inhibited the effect of LPS on PI3K, phospho-AKT and phospho-ERK1/2 expression $(P<0.05)$. Kinin+Hoe140 did not counteract the decreased expression of PI3K, phospho-AKT and phospho-ERK1/2 induced by LPS (Figure 7). These data indicate that the kallikrein/kinin system activates the PI3K/AKT and ERK1/2 signaling pathways by activating the $\mathrm{B} 2$ receptor, which may contribute to the observed anti-apoptotic effects of the kallikrein/kinin system in HEK 293 cells.

Kallikrein/kinin system inhibits apoptosis in HEK 293 cells by affecting the bcl-2 protein family

Finally, we examined the effects of the kallikrein/kinin system on the expression of different proteins in the bcl-2 protein family in HEK 293 cells using Western blotting. In nonFBS medium, LPS exposure decreased the levels of bcl-2 and bcl-xl and increased the expression of bax in HEK 293 cells. However, treatment with rAAV-HK and kinin, but not lacZ or kinin+Hoe140, attenuated the effects of LPS on the expression of the bcl-2 family of proteins (Figure 8). These data indicate that the kallikrein/kinin system directly protects against apoptosis by affecting the activity of the bcl-2 protein family.

\section{Discussion}

Accumulating evidence indicates that apoptosis is increased in
A
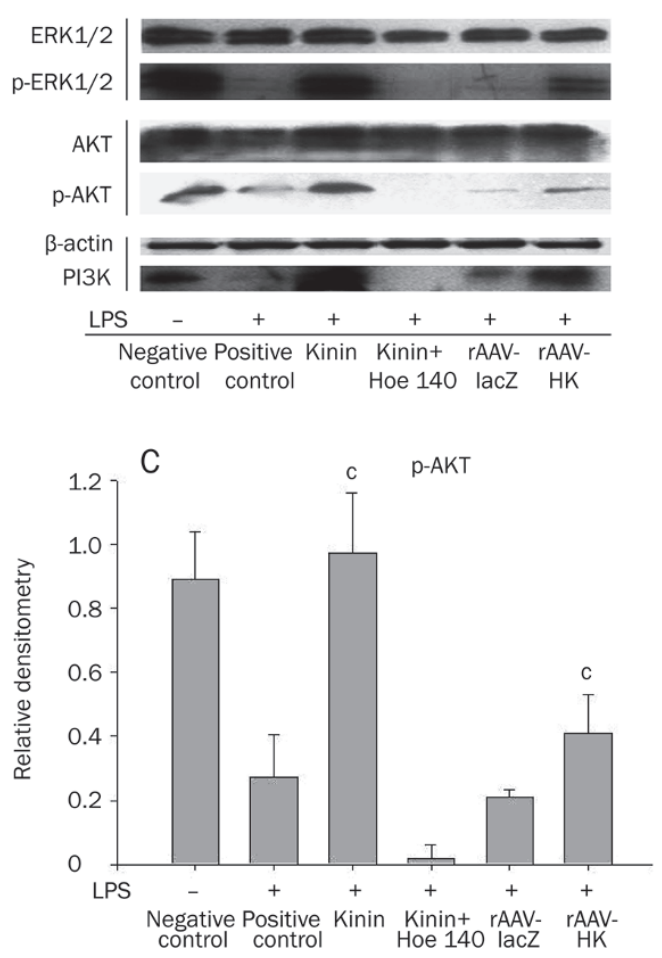
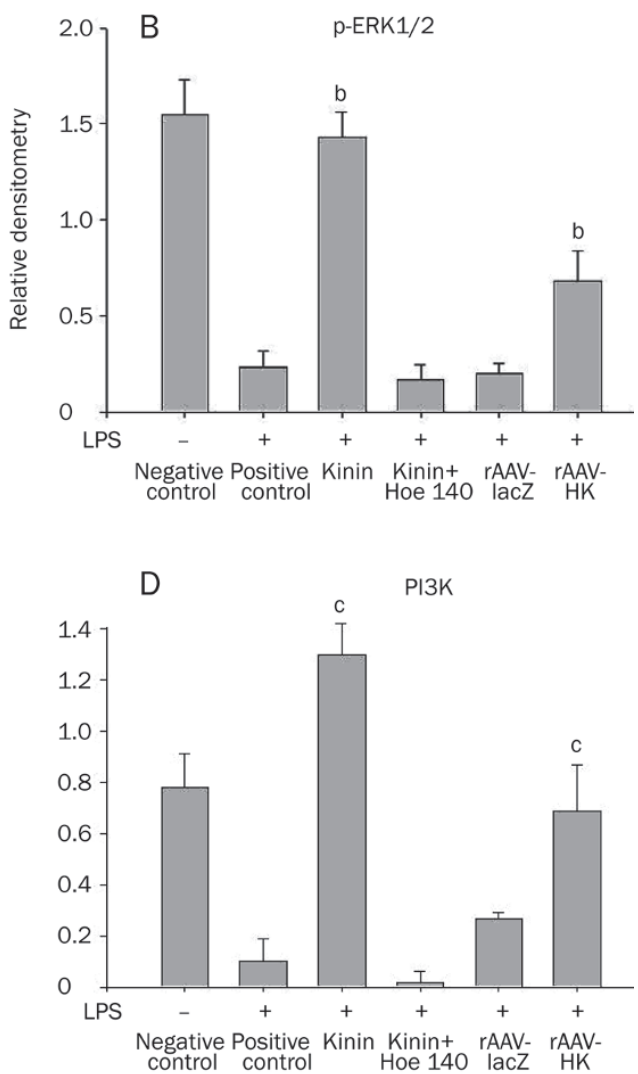

Figure 7. The kallikrein/kinin system activates cell proliferation signaling pathways in HEK 293 cells. Western blot analysis was performed on cells in non-FBS medium exposed to LPS (A), and the relative expression levels of $\mathrm{p}$-ERK1/2, P-AKT, and PI3K as determined by densitometry, were normalized to total ERK1/2, $A K T$, and $\beta$-actin, as shown in the histograms (B, C, D). The expression levels of PI3K, phospho-AKT and phospho-ERK1/2 decreased upon LPS exposure. Treatment with rAAV-HK and kinin, but not rAAVlacZ, inhibited the effect of LPS on the expression of PI3K, p-AKT and p-ERK1/2. Kinin+Hoe140 had no effects on the LPS-induced decrease in PI3K, phospho-AKT and phosphoERK1/2 expression. ${ }^{\mathrm{b}} P<0.05$, ${ }^{\mathrm{C}} P<0.01$ vs positive control. 
A
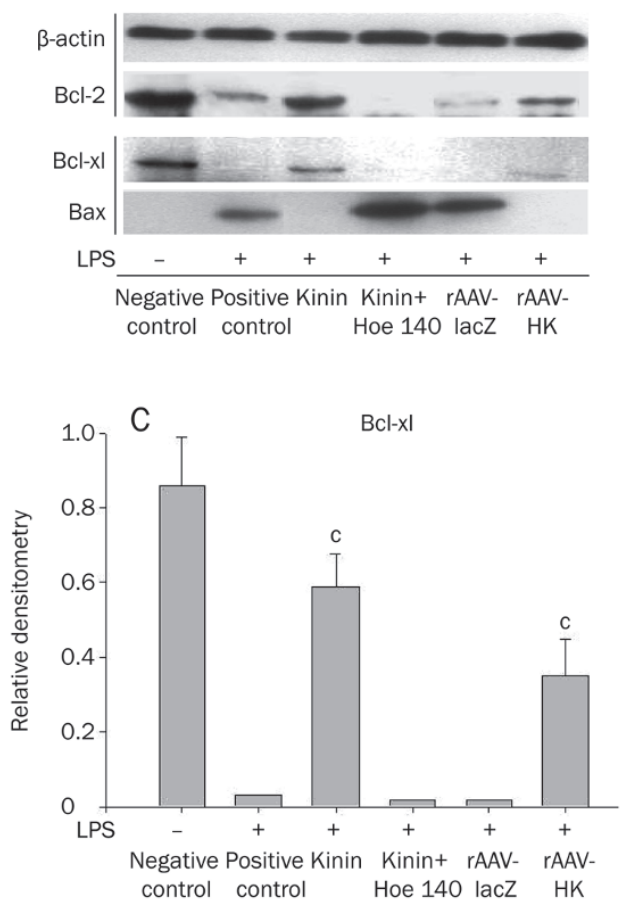
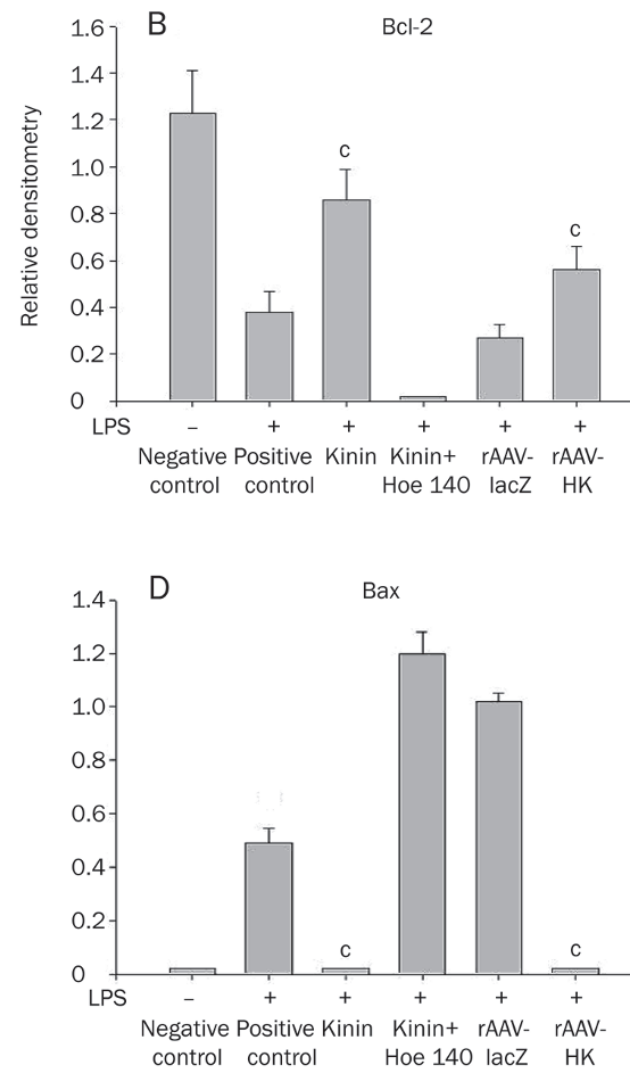

Figure 8. The kallikrein/Kinin system inhibits apoptosis by affecting the bcl-2 protein family in HEK 293 cells. Western blot analysis was performed on lysates from cells in non-FBS medium exposed to LPS (A), and the relative expression levels, as determined by densitometry, were normalized to total $\beta$-actin, as shown in the histograms $(B, C, D)$. When exposed to LPS in non-FBS medium, the protein levels of $\mathrm{bcl}-2$ and $\mathrm{bcl}-\mathrm{x}_{\mathrm{L}}$ in HEK 293 cells decreased while the protein level of bax increased. However, treatment with $\mathrm{rAAV}-\mathrm{HK}$ and kinin, but not lacZ or Kinin+Hoe140, attenuated the effects of LPS on the expression levels of the bcl-s family. ${ }^{c} P<0.01$ vs rAAV-LacZ group. the heart, kidney and aorta of hypertensive patients, resulting in heart failure, renal dysfunction and vascular remodeling ${ }^{[11]}$. The present study demonstrated that a single intravenous injection of rAAV-HK could protect against apoptosis in these organs of SHRs. Furthermore, in vitro assays showed that kallikrein/kinin activates the Bradykinin B2 receptor to inhibit LPS-induced apoptosis in HEK 293 cells. We also demonstrated that rAAV-HK modulates the expression level of the bcl-2 protein family to exert its anti-apoptotic effects, which consequently leads to decreased activation of caspase 3. In addition to modulating the apoptotic pathway, rAAV-HK also activates the PI3K/AKT and ERK1/2 pathways, which are critical to cell proliferation, both in vivo and in vitro. In summary, these data suggest that in addition to reducing blood pressure, rAAV-mediated HK gene delivery also protects against hypertension-induced apoptosis in organs affected by this chronic disease.

Increased cardiomyocyte apoptosis has been recently demonstrated in SHRs and patients with essential hypertension ${ }^{[12]}$. Although apoptosis could be induced by pressure overload, in vitro and in vivo findings indicated that angiotensin II could be critical in cardiac apoptosis ${ }^{[13,14]}$. A study of patients with essential hypertension by González and his colleagues indicated that the efficacy of antihypertensive treatment in reducing blood pressure does not predict its capacity to reduce myocardial apoptosis in hypertensive patients ${ }^{[15]}$. They also found that chronic AT1 blockade is associated with reduced myocardial apoptosis in essential hypertension ${ }^{[15]}$. The ben- eficial effects of angiotensin-converting enzyme inhibition in hypertension, cardiovascular and renal disease can be partially attributed to kinin accumulation resulting from the activation of the B2 receptor ${ }^{[16,17]}$. Previous animal studies suggested that kallikrein/kinin protects against myocardial apoptosis after ischemia/reperfusion injury ${ }^{[18,19]}$. In our study, delivery of the kallikrein gene protected against cardiomyocyte apoptosis in SHRs; therefore, this gene could potentially be used to treat hypertension.

Enhanced apoptosis is present in the kidneys of SHRs. Our previous study indicated that delivery of the kallikrein gene could attenuate renal tubule injury and decrease the level of urinary microalbumin in SHRs ${ }^{[6]}$. The present study demonstrated that kallikrein/kinin protects against hypertensive renal injury by inhibiting apoptosis in the kidney. Kallikrein/ kinin directly improves renal function, independent of its effects on blood pressure, by inhibiting apoptosis in multiple animal models, including salt-sensitive rats and gentamicininduced renal injury ${ }^{[20,21]}$. Our previous study demonstrated that rAAV-mediated HK delivery upregulates the B2 receptor and downregulates angiotension II receptor 1 , endothelin receptor $\mathrm{A}$ and vasopressin receptor $2^{[22]}$, which could also contribute to the anti-apoptotic effects of the kallikrein/kinin system in hypertensive kidney injuries.

Vascular remodeling contributes to end-organ damage in hypertension, meaning prevention of this phenomenon should be a therapeutic aim of antihypertensive therapy. Growth, apoptosis, inflammation and fibrosis all contribute to arterial 
remodeling in hypertension ${ }^{[23]}$. As shown in our study, aortas of DOCA-salt rats, SHRs and angiotensin-infused rats exhibit apoptosis ${ }^{[23]}$. Numerous inflammatory modulators are also present in the vasculature, including reactive oxygen spe$\operatorname{cies}^{[24]}, \mathrm{NO}^{[25]}$, angiotensin type 2 receptors ${ }^{[26]}$ and the endothelin system ${ }^{[27]}$. The present study suggests that kallikrein/kinin regulates apoptosis in the aorta through countervailing influences on anti-apoptotic and pro-apoptotic systems.

The present study also demonstrated that rAAV-HK or kinin delivery has a direct anti-apoptotic effect in HEK 293 cells, suggesting that the kallikrein/kinin system protects against hypertension-induced organ injury by inhibiting apoptosis and not just by lowering blood pressure.

Apoptosis is a cellular self-destruction mechanism involved in a variety of biological events. Two cellular pathways trigger apoptosis: the intrinsic pathway, for which the mitochondrion is the central organelle governed by the pro- and antiapoptotic Bcl-2 family of proteins, and the extrinsic pathway, consisting of the TNF-related family of cell surface receptors $^{[28]}$. The Bcl-2 family can be divided into the anti-apoptotic members, such as Bcl-2 and Bcl- $\mathrm{x}_{\mathrm{L}}$, and pro-apoptotic members, such as $B{ }^{[28]}$. The present study demonstrated that overexpression of the kallikrein gene in both hypertensive rats and cultured cells upregulates Bcl-2 and Bcl- $\mathrm{x}_{\mathrm{L}}$ expression and downregulates Bax expression, and the effects of kinin on the Bcl-2 family could be inhibited by a bradykinin B2 receptor inhibitor. These results suggest that kallikrein/kinin regulates the Bcl-2 protein family through the bradykinin B2 receptor to protect cells from hypertension-induced apoptosis.

The apoptotic process is executed by a family of cysteine proteases, which specifically cleave their substrates at aspartic acid residues ${ }^{[29]}$. These proteases, known as caspases, are activated through the extrinsic and/or intrinsic pathways. In the present study, delivery of the kallikrein gene decreased the activity of caspase 3, an important pro-apoptotic factor ${ }^{[29]}$, thus inhibiting apoptosis in hypertensive organs. In addition to inhibiting the apoptosis pathway, kallikrein/kinin activates the PI3K/Akt and ERK1/2 signaling pathways, which are involved in cell survival and proliferation.

In summary, delivery of the kallikrein gene protected against hypertension-induced organ damage by inhibiting cell apoptosis and enhancing cell survival. These effects were achieved by activating the PI3K/Akt and ERK1/2 signaling pathways and decreasing caspase 3 activity. This study suggests that rAAV-mediated HK gene delivery could be used as a novel hypertension treatment that not only decreases blood pressure, but also inhibits apoptosis in affected organs.

\section{Acknowledgements}

This work was supported in part by funds from the National "863" Plan Project (№ 2006AA02A406), the National Natural Science Foundation of China (№ 30800458), and the National Basic Research Program of China 973 Program (2007CB512004).

\section{Author contribution}

Dao-wen WANG designed research; Jiang-tao YAN, Tao WANG performed research; Tao WANG contributed new analytical tools and reagents; Tao WANG analyzed data; Jiang-tao YAN wrote the paper.

\section{References}

1 Chobanian AV, Bakris GL, Black HR, Cushman WC, Green LA, Izzo $\mathrm{JL}$, et al. The National High Blood Pressure Education Program Coordinating Committee. Seventh report of the joint national committee on prevention, detection, evaluation, and treatment of high blood pressure. Hypertension 2003; 42: 1206-52.

2 Phillips MI. Gene therapy for hypertension: the preclinical data. Hypertension 2001; 38: 543-8.

3 Monahan PE, Samulski RJ. AAV vectors: is clinical success on the horizon? Gene Ther 2000; 7: 24-30.

4 Mueller C, Flotte TR. Clinical gene therapy using recombinant adenoassociated virus vectors. Gene Ther 2008; 15: 858-63.

5 Chao J, Chao L. Kallikrein-kinin in stroke, cardiovascular and renal disease. Exp Physiol 2005; 90: 291-8.

6 Wang T, Li H, Zhao C, Chen C, Li J, Chao J, et al. Recombinant adeno-associated virus-mediated kallikrein gene therapy reduces hypertension and attenuates its cardiovascular injuries. Gene Ther 2004; 11: 1342-50.

7 Wang T, Hou L, Liu Z, Wang Y, Chen C, Xiao X, et al. Intramuscular delivery of raav-mediated kallikrein gene reduces hypertension and prevents cardiovascular injuries in model rats. Acta Pharmacol Sin 2007; 28: 1898-1906.

8 Yan J, Wang T, Li J, Xiao X, Wang D. Recombinant adeno-associated virus-mediated human kallikrein gene therapy prevents high-salt dietinduced hypertension without effect on basal blood pressure. Acta Pharmacol Sin 2008; 29: 808-14.

9 Xiao X, Li J, Samulski RJ. Production of high-titer recombinant adenoassociated virus vectors in the absence of helper adenovirus. J Virol 1998; 72: 2224-32.

10 Kramarenko II, Bunni MA, Morinelli TA, Raymond JR, Garnovskaya MN. Identification of functional bradykinin B2 receptors endogenously expressed in HEK293 cells. Biochem Pharmacol 2009; 77: 269-76.

11 Hamet P, Richard L, Dam TV, Teiger E, Orlov SN, Gaboury L, et al. Apoptosis in target organs of hypertension. Hypertension 1995; 26 : 642-8.

12 Gonzalez A, Fortuno MA, Querejeta R, Ravassa S, Lopez B, Lopez $\mathrm{N}$, et al. Cardiomyocyte apoptosis in hypertensive cardiomyopathy. Cardiovasc Res 2003; 59: 549-62.

13 Kajstura J, Cigola E, Malhotra A, Li P, Cheng W, Meggs LG, et al. Angiotensin II induces apoptosis of adult ventricular myocytes in vitro. J Mol Cell Cardiol 1997; 29: 859-70.

14 Diep QN, El Mabrouk M, Yue P, Schiffrin EL. Effect of at1 receptor blockade on cardiac apoptosis in angiotensin ii-induced hypertension. Am J Physiol Heart Circ Physiol 2002; 282: H1635-41.

15 Gonzalez A, Lopez B, Ravassa S, Querejeta R, Larman M, Diez J, et al. Stimulation of cardiac apoptosis in essential hypertension: potential role of angiotensin II. Hypertension 2002; 39: 75-80.

16 Messadi-Laribi E, Griol-Charhbili V, Pizard A, Vincent MP, Heudes D, Meneton $\mathrm{P}$, et al. Tissue kallikrein is involved in the cardioprotective effect of $\mathrm{AT}_{1}$-receptor blockade in acute myocardial ischemia. J Pharmacol Exp Ther 2007; 323: 210-6.

17 Westermann D, Schultheiss HP, Tschöpe C. New perspective on the tissue kallikrein-kinin system in myocardial infarction: role of angiogenesis and cardiac regeneration. Int Immunopharmacol 2008; 
8: $148-54$.

18 Yin H, Chao L, Chao J. Kallikrein/Kinin protects against myocardial apoptosis after ischemia/reperfusion via Akt-glycogen synthase kinase-3 and Akt-Bad\{middle dot\}14-3-3 signaling pathways. J Biol Chem 2005; 280: 8022-30.

19 Messadi-Laribi E, Griol-Charhbili V, Gaies E, Vincent MP, Heudes D, Meneton P, et al. Cardioprotection and kallikrein-kinin system in acute myocardial ischaemia in mice. Clin Exp Pharmacol Physiol 2008; 35: 489-93.

20 Chao J, Bledsoe G, Yin H, Chao L. The tissue kallikrein-kinin system protects against cardiovascular and renal diseases and ischemic stroke independently of blood pressure reduction. Biol Chem 2006; 387: $665-75$.

21 Bledsoe G, Crickman S, Mao J, Xia CF, Murakami H, Chao L, et al. Kallikrein/kinin protects against gentamicin-induced nephrotoxicity by inhibition of inflammation and apoptosis. Neprol Dial Transplant 2006; 21: 624-33.

22 Tu L, Xu X, Wan H, Zhou C, Deng J, Xu G, et al. Delivery of recombinant adeno-associated virus-mediated human tissue kallikrein for therapy of chronic renal failure in rats. Hum Gene Ther 2008; 19: 318-30.

23 Intengan HD, Schiffrin EL. Vascular remodeling in hypertension: Roles of apoptosis, inflammation, and fibrosis. Hypertension 2001; 38: 581-7.

24 Irani K. Oxidant signaling in vascular cell growth, death, and survival: a review of the roles of reactive oxygen species in smooth muscle and endothelial cell mitogenic and apoptotic signaling. Circ Res 2000; 87: 179-83.

25 Pollman MJ, Yamada T, Horiuchi M, Gibbons GH. Vasoactive substances regulate vascular smooth muscle cell apoptosis: countervailing influences of nitric oxide and angiotensin II. Circ Res 1996; 79: 748-56.

26 Yamada T, Horiuchi M, Dzau VJ. Angiotensin II type 2 receptor mediates programmed cell death. Proc Natl Acad Sci USA 1996; 93 : 156-60.

27 Cattaruzza M, Dimigen C, Ehrenreich H, Hecker M. Stretch-induced endothelin $B$ receptor-mediated apoptosis in vascular smooth muscle cells. FASEB J 2000; 14: 991-8.

28 Jeong SY, Seol DW. The role of mitochondria in apoptosis. BMB Rep 2008; 41: 11-22.

29 Li J, Yuan J. Caspases in apoptosis and beyond. Oncogene 2008; 27: 6194-206. 